\title{
The factors associated with hemorrhagic presentation in children with untreated brain arteriovenous malformation: a meta-analysis
}

\author{
*Xiaolin Ai, MD,' Zengpanpan Ye, MD,' Jianguo Xu, PhD, ${ }^{1}$ Chao You, PhD, ${ }^{1}$ and Yan Jiang, PhD² \\ 1Department of Neurosurgery and 2Nursing Department, West China Hospital of Sichuan University, Chengdu, Sichuan, China
}

OBJECTIVE Rupture of arteriovenous malformations (AVMs) would result in high mortality and prevalence of disability in pediatric patients. Decisions regarding the treatment of AVMs need to weigh the risk of rupture over the course of their natural history against the possibility of creating a lesion during treatment. Multiple factors have been proposed to predict hemorrhagic presentation of pediatric patients with AVMs. The aim of this meta-analysis was to evaluate the predictors of hemorrhagic presentation in pediatric patients with AVMs.

METHODS The authors searched the PubMed and EMBASE databases. Studies reporting the predictors of hemorrhagic presentation in children with untreated brain AVMs were included. The predictive ability of identified predictors was assessed by odds ratios (ORs) and 95\% confidence intervals (Cls).

RESULTS A higher risk of hemorrhagic presentation was found in AVMs with smaller size $(<3 \mathrm{~cm}, 0 \mathrm{R} 2.97,95 \% \mathrm{Cl}$ 1.94-4.54, $p<0.00001$ ), deep venous drainage (OR 2.28, 95\% Cl 1.55-3.36, $p<0.0001$ ), a single draining vein (OR $2.23,95 \% \mathrm{Cl} 1.27-3.92, \mathrm{p}=0.005)$, a single feeder (OR 3.72, 95\% Cl 1.31-10.62, $p=0.01$ ), a deep location (OR 1.82, $95 \% \mathrm{Cl} 1.22-2.72, p=0.004$ ), an infratentorial location (OR 2.25, 95\% Cl 1.19-4.26, $p=0.01$ ), and diffuse morphology (OR 8.94, 95\% Cl 3.01-26.55, $\mathrm{p}<0.0001)$. In addition, the AVMs with draining vein ectasia (OR 0.35, 95\% $\mathrm{Cl} 0.13-0.97$, $p=0.04$ ) and high Spetzler-Martin (SM) grade (OR 0.53, 95\% Cl 0.36-0.78, $p=0.001$ ) had a lower risk of hemorrhagic presentation in pediatric patients.

CONCLUSIONS Smaller AVMs, deep venous drainage, a single draining vein, a single feeder, deep/infratentorial location, diffuse morphology, and high SM grade were identified as positive predictors for hemorrhagic presentation. Particularly, patients with diffuse AVMs have a higher risk of hemorrhagic presentation than other factors and may need active treatments. However, factors such as age, sex, draining vein stenosis, and associated aneurysms were not associated with hemorrhagic presentation.

https://thejns.org/doi/abs/10.3171/2018.9.PEDS18262

KEYWORDS arteriovenous malformation; pediatric; hemorrhagic presentation; vascular disorders

$\mathrm{B}$ RAIN arteriovenous malformations (AVMs) are the most common cause of intracranial hemorrhage in pediatric patients. ${ }^{34}$ AVM rupture in pediatric patients leads to a high mortality rate of $25 \%$ and permanent neurological defects in $20.2 \%-40.6 \%$ of patients. ${ }^{7,26,39}$ Decisions regarding the treatment of AVMs need to weigh the risk of hemorrhage over the course of their natural history against the possibility of creating a lesion during treatment. Despite the remarkable improvement in the treatment of AVMs, the natural history and risk factors of pediatric AVMs remain unclear. ${ }^{17}$
The angioarchitecture, clinical presentation, and outcomes of AVMs are different in pediatric and adult patients. The annual hemorrhagic rate of AVMs in the general population ranges from $2 \%$ to $4.61 \%,{ }^{10,19}$ whereas the risk of hemorrhage in pediatric patients is not entirely understood. AVM rupture is more common in the pediatric population than in the adult population and accounts for $58 \%-77 \%$ of total admissions. ${ }^{83}$ In addition, many studies $^{29,35}$ reported that AVMs in children more often have exclusively deep venous drainage and are located in deep areas of the brain, both of which would increase the risk

ABBREVIATIONS AVM = arteriovenous malformation; $\mathrm{Cl}=$ confidence interval; NOS = Newcastle-Ottawa Scale; OR = odds ratio; $\mathrm{SM}=$ Spetzler-Martin . SUBMITTED May 4, 2018. ACCEPTED September 6, 2018.

${ }^{*}$ X.A. and Z.Y. contributed equally to this work. 
of intracranial hemorrhage. Multiple factors have been proposed as predictors for hemorrhagic presentation among pediatric patients with AVMs, ${ }^{17,59}$ such as size, draining vein, location, associated aneurysm, and nidal morphology.

A previous meta-analysis ${ }^{37}$ had focused on AVM rupture risk in adult patients, and only adult patients were included in the cohort of the Scottish Intracranial Vascular Malformation Study (SIVMS) and Multicenter AVM Research Study (MARS). In addition, the mean age of the included patients was about 37 years, which may not reflect the clinical characteristic of pediatric patients. However, pediatric patients with AVMs differ from adult patients in terms of the clinical characteristics of the AVMs, ${ }^{3,17}$ clinical presentations, and outcomes. ${ }^{83}$ Thus, for hemorrhagic presentation, we assessed the rate of hemorrhage and risk factors in pediatric patients using a meta-analysis to identify better treatment for pediatric patients with AVMs.

\section{Methods \\ Literature Review}

This meta-analysis was performed according to the Preferred Reporting Items for Systematic Reviews and Meta-Analyses (PRISMA) guidelines (Fig. 1). We searched the literature from the beginning of indexing until April 3, 2018, using the Cochrane Central Register of Controlled Trials, PubMed, and EMBASE databases. The following words were used: ("cerebral arteriovenous malformations" OR "brain arteriovenous malformations") AND ("children" OR "pediatric"). The detailed search strategy was as follows: (“"child”[MeSH Terms] OR “child”[All Fields] OR “children”[All Fields]) OR ("pediatrics"[MeSH Terms] OR "pediatrics"[All Fields] OR "pediatric"[All Fields])) AND ((“intracranial arteriovenous malformations"[MeSH Terms] OR ("intracranial"[All Fields] AND "arteriovenous"[All Fields] AND "malformations"[All Fields]) OR "intracranial arteriovenous malformations"[All Fields] OR ("cerebral"[All Fields] AND "arteriovenous"[All Fields] AND "malformations" [All Fields]) OR "cerebral arteriovenous malformations"[All Fields]) OR ((“brain”[MeSH Terms] OR "brain"[All Fields]) AND ("arteriovenous malformations"[MeSH Terms] OR ("arteriovenous"[All Fields] AND "malformations”[All Fields]) OR “arteriovenous malformations"[All Fields]))). The additional related studies were found by checking the references of retrieved articles.

\section{Inclusion and Exclusion Criteria}

After filtering duplicates, the titles and abstracts of all available articles were screened to find the relevant articles. The full texts of these articles were independently reviewed by two reviewers, following the inclusion and exclusion criteria. The inclusion criteria were as follows: 1) articles reporting pediatric patients (age $<18$ years) diagnosed with cerebral AVMs; 2) articles reporting the odds ratio (OR) and $95 \%$ confidence interval $(\mathrm{CI})$ of variables for hemorrhagic presentation; and 3) randomized controlled studies, and cohort, case-control, or case reports ( $>$ 20 cases). If the OR was $>1$, the factors were considered as increasing the risk of hemorrhage of untreated AVMs, and the factors with ORs $<1$ were considered as having protective effects. The original data could be used to calculate the ORs and 95\% CIs as follows: OR =ad/bc, in which "a" = number of patients with hemorrhage and the factor, " $b$ " = number of patients with the factor but without hemorrhage, "c" = number of patients with hemorrhage but without the factor, and "d" = number of patients without hemorrhage and the factor. The exclusion criteria were as follows: 1) articles not in English, 2) systematic reviews or animal articles, and 3) patients who had previously undergone treatment for an AVM.

\section{Data Extraction}

The data from included articles were extracted independently by two reviewers, and the disagreement was resolved by the third reviewer. With a standard data form, the following data were extracted: author, year of publication, country, study design, number of patients, sex, mean age of patients on admission, hemorrhage rate, mean score of Spetzler-Martin (SM) grade, study period, NewcastleOttawa Scale (NOS) ${ }^{73}$ and the ORs and $95 \%$ CIs of variables for hemorrhagic presentation. AVM was diagnosed by digital subtraction angiography, magnetic resonance angiography, or CT angiography. Hemorrhagic presentation was the initial presentation at diagnosis that was confirmed with CT or MRI. The results of multivariate regression analysis were pooled for analysis when they were available; if not, the results of univariate regression analysis were pooled.

\section{Quality Assessment of the Included Articles}

Two reviewers evaluated the quality of each included study independently using the NOS ${ }^{73}$ which ranged from 0 to 9 . A high-quality study was defined as one with an NOS score $\geq 6$, and a low-quality study as one with an NOS score $<6$.

\section{Data Analysis}

The risk of variables for hemorrhagic presentation was evaluated by the pooled ORs and $95 \%$ CIs. The risk of variables for hemorrhagic presentation was pooled by Review Manager (version 5.3, Cochrane Collaboration). By the generic inverse variance method, the pooled results of discontinuous variables were presented as ORs with 95\% CIs. The pooled results of continuous variables were presented as mean differences with $95 \%$ CIs. The factors were categorized as having minimal evidence (OR 1.01.5 or $0.9-1.0)$, moderate evidence (OR $1.5-2.0$ or $0.8-$ 0.9 ), and strong evidence (OR $>2.0$ or $<0.8)$. According to the Cochrane review guidelines ${ }^{31}$ the heterogeneity of the pooled outcomes was assessed by the $\mathrm{I}^{2}$ statistic. If $\mathrm{I}^{2}$ was $<50 \%$, the heterogeneity was not significant and the fixed-effects model was used. If $\mathrm{I}^{2}$ was $>50 \%$, heterogeneity was significant and the random-effects model was used. ${ }^{32}$ The sensitivity analysis was used to confirm the robustness of pooled results. ${ }^{56}$ If the number of articles referring to the same risk factor was more than 5, the publication bias of included articles was assessed by a funnel plot with Begg's rank correlation, using Stata (version 


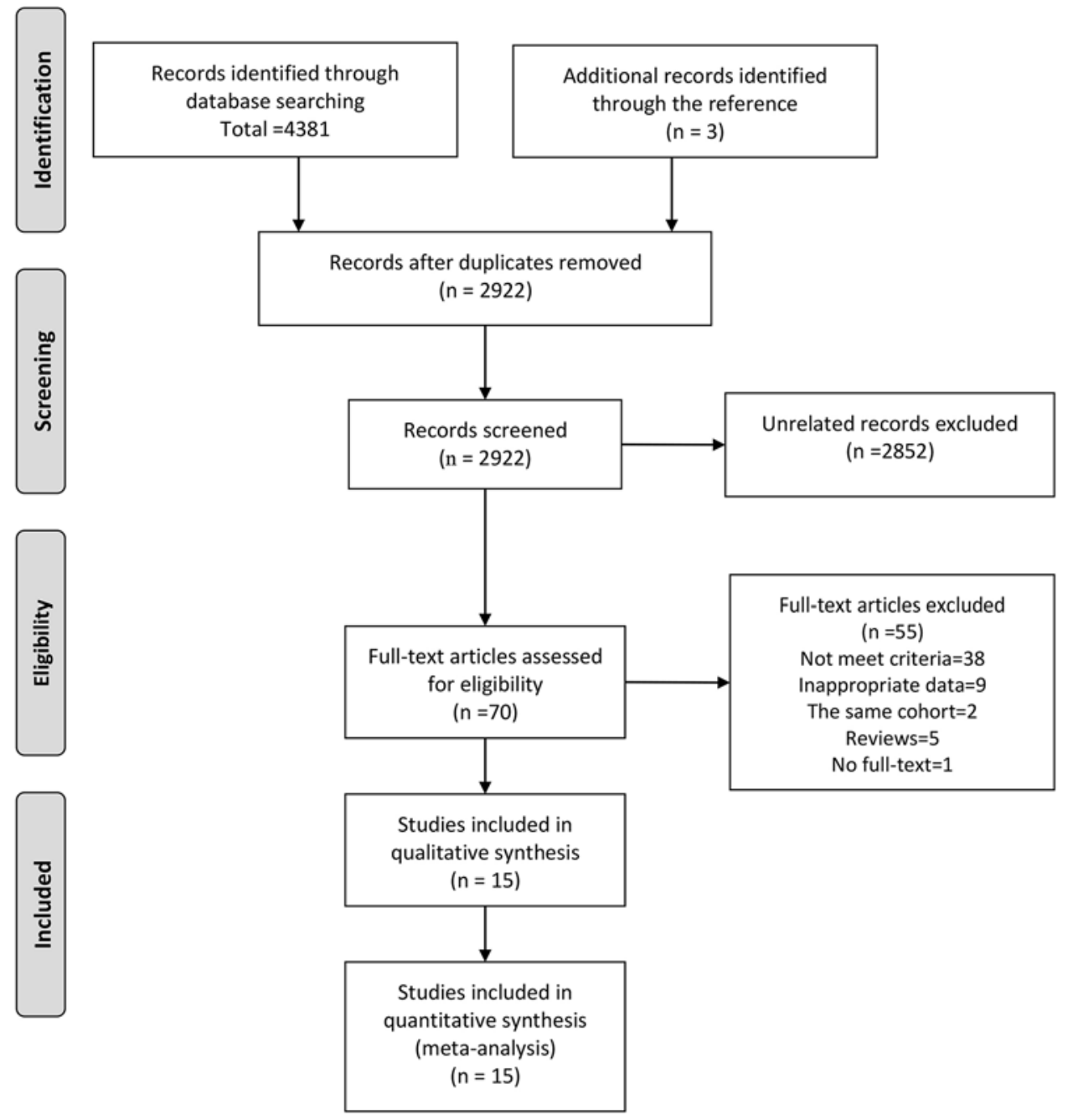

From: Moher D, Liberati A, Tetzlaff J, Altman DG, The PRISMA Group (2009). Preferred Reporting /tems for Systematic Reviews and MetaAnalyses: The PRISMA Statement. PLoS Med 6(7): e1000097. doi:10.1371/journal.pmed1000097

FIG. 1. The PRISMA flow diagram of procedures to search the included studies.

13.0, StataCorp.). The statistical significance was defined as $\mathrm{p}<0.05$.

\section{Results}

\section{Literature Search}

Initial research identified 70 eligible articles and included 15 articles ${ }^{1,2,11,16,17,20,35,46,47,51,53,59,62,66,76}$ that described any factors associated with hemorrhagic presentation, after excluding 55 articles for the following reasons: no data for calculating the risk of variables for hemorrhagic presentation, $22,44,58,63,64,75,79,83$ inclusion of the same cohort as another report, ${ }^{48,69}$ patients with AVMs treated before hemorrhage, ${ }^{14,49,51,55,74}$ inclusion of only specific cases (such as patients with hereditary hemorrhagic telangiectasia ${ }^{78}$ cerebellar AVM, ${ }^{25}$ Gamma Knife surgery, ${ }^{4,6-8,13,15,24,27,34,}$ $38,40,54,57,60,70,80-82$ embolization, ${ }^{12,67}$ or microsurgery ${ }^{18,23,26,43}$ treatment), impossibility of separating pediatric patients from adult patients, ${ }^{10,28,33,41,45,77}$ less than 20 cases included, ${ }^{65}$ reviews, ${ }^{5,21,40,52,61}$ or full text unavailable..$^{50}$

\section{Characteristics of Included Studies}

A total of 15 articles with 1155 pediatric patients were included in this meta-analysis, including 2 prospective $\operatorname{articles}^{11,46}$ and 13 retrospective articles ${ }^{1,2,16,17,20,35,47,51,53,59 \text {, }}$ $62,66,76$ (Table 1). All the included studies were conducted from 2006 to 2018. The mean age of the patients ranged from 10.1 to 13.4 years and the percentage of patients with first presentation with hemorrhage ranged from $41 \%$ to $78 \%$. The median SM grade ranged from 2 to 3 . All included studies were high quality, with scores $\geq 6$ on the NOS. Among the 15 included articles, the ORs of 14 risk factors were extracted for analysis, including age, sex, size of AVM, deep venous drainage, single draining vein, draining vein ectasia/stenosis, single feeder, eloquent location, deep location, infratentorial location, associated aneurysm, nidal morphology (diffuse AVMs: presence of significant intervening brain within the AVM nidus; compact AVMs: presence of little or no intervening brain within the AVM nidus), ${ }^{17}$ and high SM grade (4-5). 
TABLE 1. Characteristics of included articles

\begin{tabular}{lllrlrrrrr}
\hline \multicolumn{1}{c}{ Authors \& Year } & Country & Study Design & $\begin{array}{c}\text { No. of } \\
\text { Pts }\end{array}$ & $\begin{array}{c}\text { Sex } \\
\text { (M/F) }\end{array}$ & $\begin{array}{c}\text { Mean Age } \\
\text { (yrs) }\end{array}$ & $\begin{array}{c}\text { Hemorrhage } \\
\text { Rate (\%) }\end{array}$ & $\begin{array}{c}\text { Median SM } \\
\text { Grade (IQR) }\end{array}$ & $\begin{array}{c}\text { Study } \\
\text { Period }\end{array}$ & $\begin{array}{c}\text { NOS } \\
\text { Score }\end{array}$ \\
\hline Oulasvirta et al., 2018 & Finland & Retro & 127 & $68: 59$ & 12.0 & $95(75)$ & $3(2)$ & $1942-2014$ & 7 \\
\hline Ma et al., 201748 & China & Prospective & 110 & $72: 38$ & 10.9 & $52(47)$ & $2(2)$ & $2009-2015$ & 8 \\
\hline Yang et al., 2016 & US & Retro & 90 & $39: 51$ & 13.3 & $37(41)$ & $3(2)$ & $1990-2013$ & 7 \\
\hline Reitz et al., 2016 & Germany & Retro & 46 & $24: 22$ & 12.4 & $31(67)$ & $2(2)$ & $1992-2015$ & 8 \\
\hline Nerva et al., 2016 & US & Retro & 40 & $26: 14$ & 11.5 & $27(68)$ & $3(2)$ & $2005-2012$ & 7 \\
\hline Abecassis et al., 2016 & US & Retro & 26 & $19: 7$ & 12.5 & $17(65)$ & $3(2)$ & $2005-2012$ & 6 \\
\hline Ma et al., 2015 & China & Retro & 108 & $43: 65$ & 10.9 & $66(61)$ & - & $2009-2014$ & 7 \\
\hline Fok et al., 2015 & China & Retro & 67 & $28: 39$ & 12.0 & $52(78)$ & - & $2005-2013$ & 8 \\
\hline Kellner et al., 2014 & US & Retro & 81 & $43: 38$ & 13.3 & $54(63)$ & $2(2)$ & $1991-2012$ & 7 \\
\hline Ellis et al., 2013 & US & Retro & 135 & $70: 65$ & 10.1 & $86(64)$ & - & $2000-2011$ & 7 \\
\hline Anderson et al., 2012 & US & Retro & 77 & $38: 39$ & 13.4 & $48(63)$ & $2(2)$ & $1991-2010$ & 6 \\
\hline Skjøth-Rasmussen et al., 2012 & Denmark & Retro & 40 & $23: 17$ & 11.3 & $28(68)$ & $3(2)$ & $2000-2008$ & 7 \\
\hline Darsaut et al., 2011 & US & Prospective & 120 & $61: 59$ & 11.7 & $58(69)$ & $3(2)$ & $1985-2009$ & 8 \\
\hline Dorfer et al., 2010 & Austria & Retro & 56 & $26: 30$ & 10.9 & $36(64)$ & $2(2)$ & $1998-2008$ & 7 \\
\hline Sanchez-Mejia et al., 2006 & US & Retro & 32 & $21: 11$ & 11.9 & $19(59)$ & $2(2)$ & $1997-2003$ & 7 \\
\hline
\end{tabular}

$I Q R=$ interquartile range; $p t s=$ patients; retro $=$ retrospective.

\section{Risk Factors for Hemorrhagic Presentation} Hemorrhagic Rate

All 15 articles reported the hemorrhage rate of AVMs, and the probability of hemorrhagic presentation accounted for $63 \%\left(95 \%\right.$ CI $\left.58 \%-69 \%, \mathrm{I}^{2}=72.2 \%\right)$ of pediatric AVMs.

\section{Age on Admission}

Among the included articles, seven $n^{1,2,47,51,62,66,76}$ provided the mean age of patients with ruptured and unruptured AVMs, and five ${ }^{1,11,59,62,66}$ provided data to calculate the risk of age $<12$ years for hemorrhagic presentation. The pooled results showed that the age of patients in the ruptured AVM group (242 cases) was significantly lower than that in the unruptured AVM group (171 cases), with a weighted mean difference of $-1.53\left(95 \% \mathrm{CI}-2.34\right.$ to $-0.72, \mathrm{p}=0.0002, \mathrm{I}^{2}$ $=49 \%$; Fig. 2A). However, the pooled results of patients < 12 years old for hemorrhagic presentation suggested age was not associated with hemorrhagic presentation (OR $1.38,95 \%$ CI $0.47-5.05, \mathrm{p}=0.55$; Fig. 2B), with a randomeffects model for the significant heterogeneity of 5 articles $\left(\mathrm{p}=0.03\right.$ and $\left.\mathrm{I}^{2}=62 \%\right)$. Based on sensitivity analysis, the heterogeneity was attributed to the article by Skjøth-Rasmussen et al. ${ }^{66}$ After excluding this article, there was no significant heterogeneity $\left(\mathrm{p}=0.37\right.$ and $\left.\mathrm{I}^{2}=4 \%\right)$ and no association between age and hemorrhagic presentation was found (OR $0.81,95 \%$ CI $0.42-1.56, \mathrm{p}=0.53$ ).

\section{Sex}

In 11 articles with 826 cases, two ${ }^{11,46}$ showed that there was no relationship between sex and hemorrhagic presentation with multivariate regression, and 10 articles $^{1,2,11,16,47}$, $51,53,62,66,76$ analyzed the association between sex and hemorrhage using univariate regression. There was no heterogeneity among the results of these articles $\left(\mathrm{p}=0.60\right.$ and $\mathrm{I}^{2}$
$=0 \%$ ) and the fixed-effects model was used. The pooled results suggested that sex had no association with hemorrhagic presentation, with an OR of 0.95 (95\% CI 0.76-1.19, $\mathrm{p}=0.68$; Fig. 2C).

\section{Deep Venous Drainage}

Among the 11 articles that reported the relationship between deep venous drainage and hemorrhagic presentation, four $17,35,53,59$ analyzed the association using multivariate regression and seven ${ }^{2,11,20,46,51,62,76}$ used univariate regression. There was no significant heterogeneity among these articles $\left(p=0.08\right.$ and $\left.\mathrm{I}^{2}=40 \%\right)$ and the fixed-effects model was used. The pooling results suggested that deep venous drainage was associated with the hemorrhagic presentation of AVMs (OR 2.28, 95\% CI 1.55-3.36, p < 0.0001; Fig. 3A).

\section{Single Draining Vein}

Three articles ${ }^{17,20,59}$ compared the risk of hemorrhage with a single draining vein and multiple draining veins $(\geq 2)$. No heterogeneity was found among these articles, and a fixed-effects model was used $\left(\mathrm{p}=0.36\right.$ and $\left.\mathrm{I}^{2}=3 \%\right)$. After pooling the results, we found that a single draining vein was associated with a higher risk of hemorrhagic presentation (OR 2.23, 95\% CI 1.27-3.92, p = 0.005; Fig. 3B).

\section{Draining Vein Ectasia}

Four articles ${ }^{17,20,47,59}$ reported the association between draining vein ectasia and hemorrhagic presentation. All articles provided the data to calculate the risk by univariate regression. There was no significant heterogeneity among these articles $\left(p=0.27\right.$ and $\left.\mathrm{I}^{2}=23 \%\right)$. By the fixed-effects model, the pooled results suggested that draining vein ectasia was associated with a lower risk of hemorrhage (OR $0.35,95 \%$ CI 0.13-0.97, $p=0.04$; Fig. 3C). 


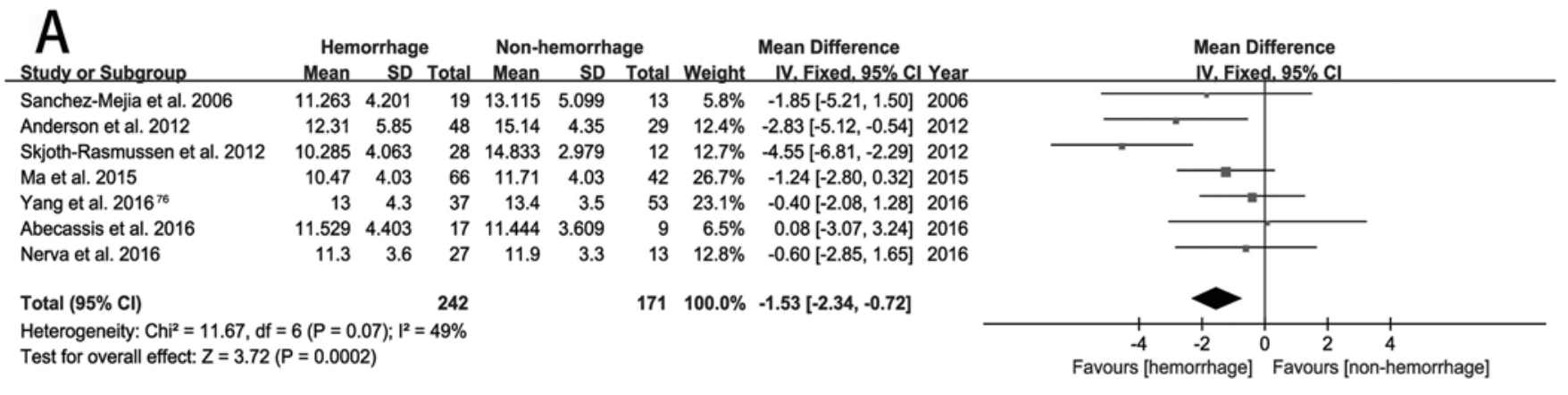

\begin{tabular}{lrrr} 
B & & & \\
Study or Subgroup & log[Odds Ratio] & SE & Weight \\
\hline Abecassis et al. 2016 & -0.3857 & 0.8442 & $18.5 \%$ \\
Darsaut et al. 2011 & -0.821 & 0.5161 & $25.6 \%$ \\
Reitz et al. 2016 & 0.4121 & 0.6907 & $21.7 \%$ \\
Sanchez-Mejia et al. 2006 & 0.4947 & 0.7597 & $20.2 \%$ \\
Skjoth-Rasmussen et al. 2012 & 2.9857 & 1.1164 & $13.9 \%$ \\
& & & \\
Total (95\% Cl) & & & $100.0 \%$ \\
Heterogeneity: Tau $^{2}=0.91 ;$ Chi $^{2}=10.65$, df $=4(P=0.03) ; I^{2}=62 \%$ \\
Test for overall effect: $Z=0.59(P=0.55)$
\end{tabular}

Odds Ratio Odds Ratio Random. $95 \% \mathrm{Cl}$ $0.68[0.13,3.56]$ $0.44[0.16,1.21]$ $1.51[0.39,5.85]$ $1.64[0.37,7.27]$ $19.80[2.22,176.59]$

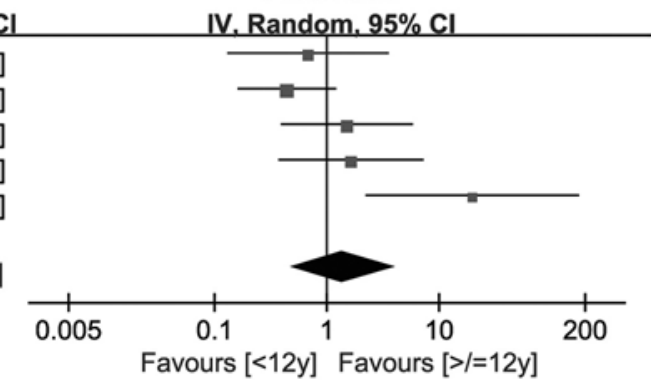

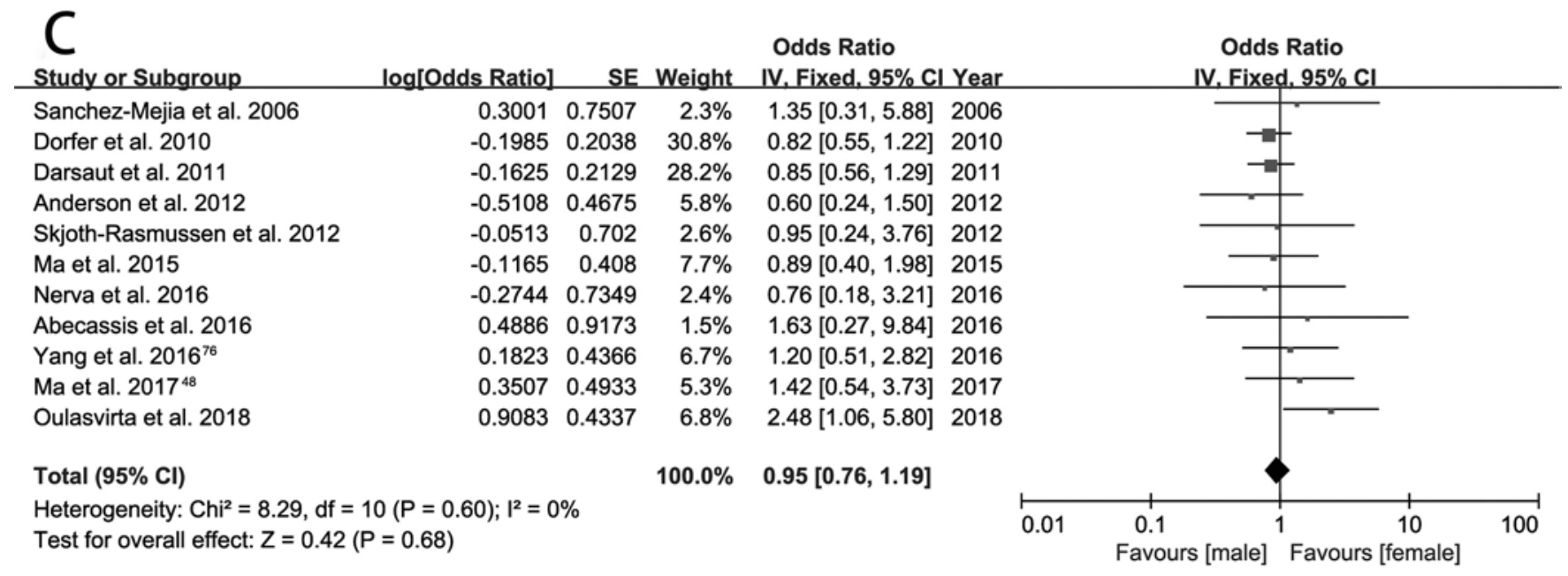

FIG. 2. Forest plots for comparison of epidemiology, including mean age (A), age > 12 years (B), and sex (C), in the hemorrhage and nonhemorrhage groups. IV = inverse variance methods.

\section{Draining Vein Stenosis}

Three articles ${ }^{172,20,59}$ provided the number of patients with draining vein stenosis. One article ${ }^{59}$ reported that hemorrhage presented in all 3 patients with draining vein stenosis, and the risk could not be calculated. Thus, we analyzed the risk of draining vein stenosis with the number of patients and found that draining vein stenosis had no effect on the rate of hemorrhagic presentation (OR 1.27, $95 \%$ CI $0.56-2.85, \mathrm{p}=0.57$; Fig. 3D). No significant heterogeneity was found among these articles $(\mathrm{p}=0.24$ and $\left.\mathrm{I}^{2}=31 \%\right)$.

\section{Single Feeder}

Only 2 studies ${ }^{20,59}$ compared the risk of hemorrhagic presentation in AVMs with a single feeder and multiple feeders $(\geq 2)$. There was no heterogeneity between the 2 articles $\left(\mathrm{p}=0.39\right.$ and $\left.\mathrm{I}^{2}=0 \%\right)$, and the fixed-effects model was used. The pooled results suggested that the single feeder increased the rate of hemorrhage compared with multiple feeders (OR 3.72, 95\% CI 1.31-10.62, $\mathrm{p}=0.01$; Fig. 3E).

\section{AVM Size}

Ten articles ${ }^{2,11,17,20,46,51,53,59,66,76}$ reported the association between the AVM size and hemorrhagic presentation. Among these articles, seven ${ }^{2,11,17,20,53,59,66}$ compared the risk of hemorrhage in larger $(\geq 3 \mathrm{~cm})$ and smaller $(<3$ $\mathrm{cm})$ AVMs. Three articles ${ }^{46,51,76}$ reported the risk of AVM size for hemorrhage, but did not define the cutoff values for AVM size. After pooling the results of 7 articles, we 
A

Odds Ratio

Odds Ratio

Study or Subgroup log[Odds Ratio] SE Weight

IV. Fixed. $95 \% \mathrm{Cl}$ Year IV. Fixed, $95 \%$ CI

Sanchez-Mejia et al. 2006

0.69310 .7281

$1.1249 \quad 0.6815$

$\begin{array}{lll}0.4121 & 0.4795\end{array}$

$7.4 \%$

$2.00[0.48,8.33] 2006$

Anderson et al. 2012

$1.5974 \quad 0.6811$

$8.4 \%$

Ellis et al. 2013

$2.1066 \quad 0.9331$

$1.51[0.59,3.86] 2012$

Kellner et al. 2014

$0.7793 \quad 0.6498$

$\begin{array}{ll}1.0152 & 0.8138\end{array}$

$\begin{array}{ll}0.571 & 0.4313\end{array}$

Yang et al. $2016^{76}$

$\begin{array}{rr}-1.0217 & 0.7073\end{array}$

$\begin{array}{lll}3.3925 & 1.2737\end{array}$

$\begin{array}{ll}1.5864 & 0.7075\end{array}$

$4.5 \% \quad 8.22[1.32,51.18] 2014$

$9.3 \% \quad 2.18[0.61,7.79] 2015$

$5.9 \% \quad 2.76[0.56,13.60] 2015$

$21.0 \% \quad 1.77[0.76,4.12] 2016$

$7.8 \% \quad 0.36[0.09,1.44] 2016$

$2.4 \% 29.74[2.45,361.01] 2016$

Oulasvirta et al. 2018

$7.8 \% \quad 4.89[1.22,19.55] 2018$

Total $(95 \% \mathrm{Cl})$

$100.0 \% \quad 2.28[1.55,3.36]$

Heterogeneity: $\mathrm{Chi}^{2}=16.59, \mathrm{df}=10(\mathrm{P}=0.08) ; \mathrm{I}^{2}=40 \%$

Test for overall effect: $Z=4.16(P<0.0001)$

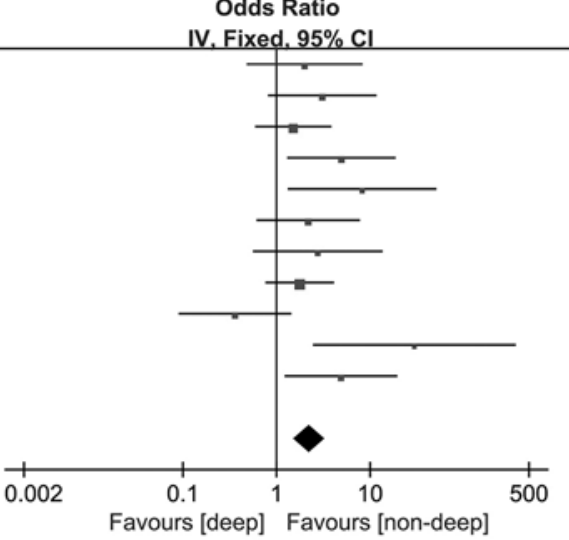

B

Odds Ratio

Odds Ratio

Study or Subgroup log[Odds Ratio] SE Weight IV. Fixed. 95\% Cl IV. Fixed, $95 \% \mathrm{Cl}$

Ellis et al. 2013

$\begin{array}{llll}0.6419 & 0.3645 & 62.0 \% & 1.90\end{array}[0.93,3.88]$

$\begin{array}{llll}0.6043 & 0.5951 & 23.2 \% & 1.83[0.57,5.87]\end{array}$

Fok et al. 2015

1.79180 .7462

$14.8 \% \quad 6.00[1.39,25.90]$

Total $(95 \% \mathrm{Cl})$

$100.0 \% \quad 2.23[1.27,3.92]$

Heterogeneity: $\mathrm{Chi}^{2}=2.06, \mathrm{df}=2(\mathrm{P}=0.36) ; \mathrm{l}^{2}=3 \%$

Test for overall effect: $Z=2.80(P=0.005)$

\section{C}

Odds Ratio

Study or Subgroup log[Odds Ratio] SE Weight IV. Fixed. 95\% CI

Ellis et al. 2013

Fok et al. 2015

Ma et al. $2017^{48}$

$-0.16250 .9202$

$32.1 \%$

$0.85[0.14,5.16]$

$-1.42710 .9142$

$-2.83091 .2075$

$-0.03051 .2731$

$32.5 \% \quad 0.24[0.04,1.44]$

$18.6 \% \quad 0.06[0.01,0.63]$

$16.8 \% \quad 0.97[0.08,11.76]$

$100.0 \% \quad 0.35[0.13,0.97]$

Heterogeneity: $\mathrm{Chi}^{2}=3.92, \mathrm{df}=3(\mathrm{P}=0.27) ; \mathrm{I}^{2}=23 \%$

Test for overall effect: $Z=2.01(P=0.04)$
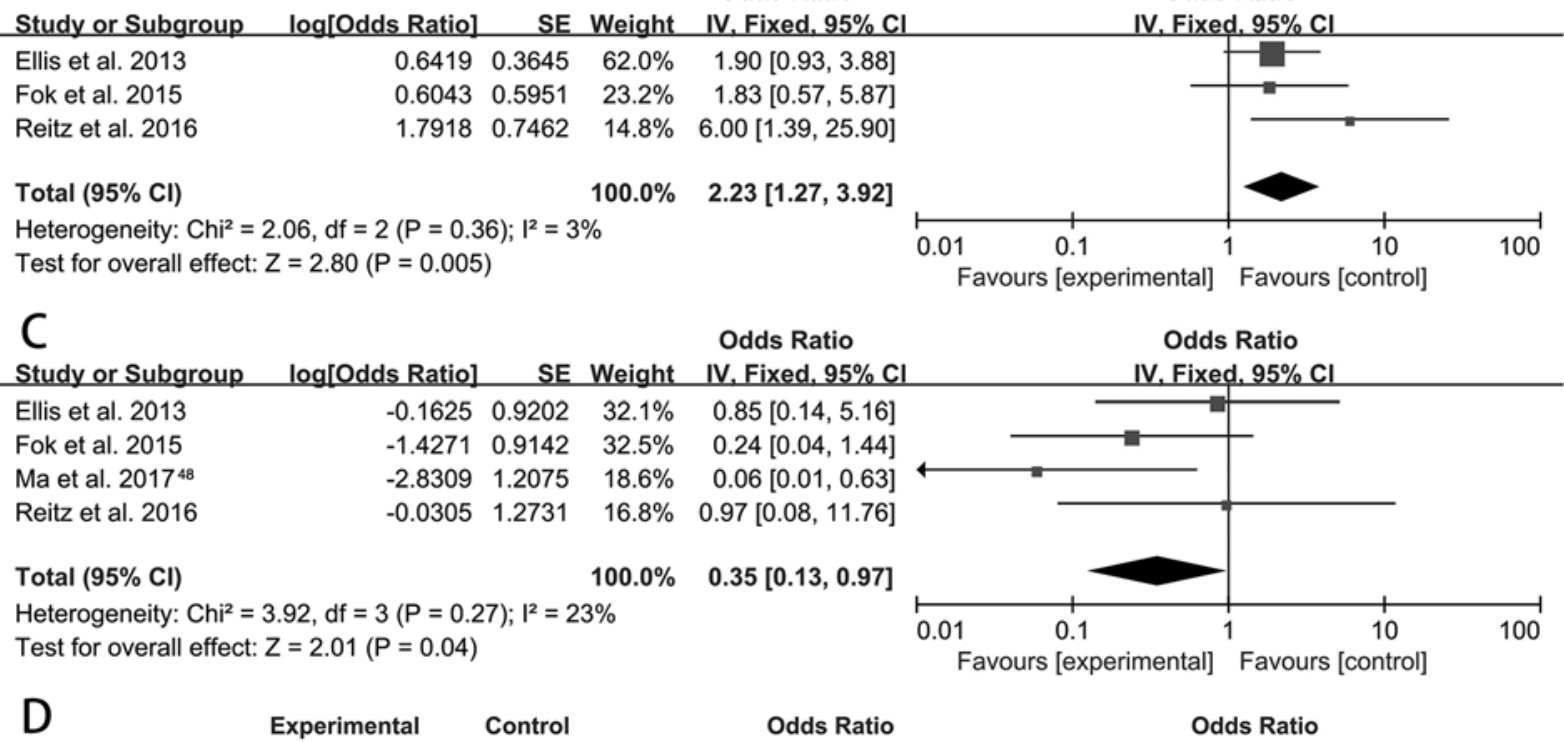

Experimental

Control

Odds Ratio

Study or Subgroup

Events Total

Events

M-H, Fixed, 95\% Cl

Ellis et al. 2013

$\begin{array}{lllll}3 & 3 & 28 & 43 & 5.5 \%\end{array}$

$3.81[0.18,78.56]$

Fok et al. 2015

$\begin{array}{lllll}2 & 4 & 50 & 63 & 27.8 \%\end{array}$

$0.26[0.03,2.03]$

Reitz et al. 2016

$17 \quad 24$

$69 \quad 111 \quad 66.7 \%$

$1.48[0.57,3.86]$

Total $(95 \% \mathrm{CI})$

31

$217100.0 \%$

$1.27[0.56,2.85]$

Total events $22 \quad 147$

Heterogeneity: $\mathrm{Chi}^{2}=2.89, \mathrm{df}=2(\mathrm{P}=0.24) ; \mathrm{I}^{2}=31 \%$

Test for overall effect: $Z=0.58(P=0.57)$

E

Odds Ratio

Study or Subgroup log[Odds Ratio] SE Weight IV. Fixed, $95 \% \mathrm{Cl}$

Fok et al. 2015

$0.7747 \quad 0.8259$ $1.7047 \quad 0.7018$

$41.9 \% \quad 2.17[0.43,10.95]$

Reitz et al. 2016 $0 \%$

Total $(95 \% \mathrm{Cl})$

Heterogeneity: $\mathrm{Chi}^{2}=0.74, \mathrm{df}=1(\mathrm{P}=0.39) ; \mathrm{l}^{2}=0 \%$

Test for overall effect: $Z=2.46(P=0.01)$

$58.1 \% 5.50[1.39,21.76]$

$00.0 \% 3.72[1.31,10.62]$

FIG. 3. Forest plots for association of hemorrhagic presentation and draining vein of AVMs, including deep venous drainage (A), single draining vein (B), draining vein ectasia (C), draining vein stenosis (D), and single feeder (E). M-H = Mantel-Haenszel. 
A

\begin{tabular}{l} 
Study or Subgroup \\
\hline Darsaut et al. 2011 \\
Anderson et al. 2012 \\
Skjoth-Rasmussen et al. 2012 \\
Ellis et al. 2013 \\
Fok et al. 2015 \\
Reitz et al. 2016 \\
Oulasvirta et al. 2018
\end{tabular}

Oulasvirta et al. 2018

Total $(95 \% \mathrm{Cl})$

Heterogeneity: $\mathrm{Ch}^{2}=9.64, \mathrm{df}=6(\mathrm{P}=0.14) ; \mathrm{I}^{2}=38 \%$

Test for overall effect: $Z=5.02(P<0.00001)$

\section{B}

Study or Subaroup log[Odds Ratio] SE Weight IV. Fixed.95\%

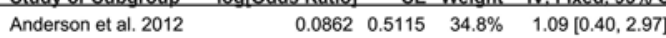

Darsaut et al. 2011

Nerva et al. 2016

Yang et al. 2016 $\begin{array}{llll}0.9361 & 1.0919 & 7.6 \% & 2.55[0.30,21.68\end{array}$

$\begin{array}{llll}0.5306 & 0.6781 & 19.8 \% & 1.70[0.45,6.42\end{array}$

Total $(95 \% \mathrm{Cl})$

Heterogeneity: $\mathrm{Chi}^{2}=0.66, \mathrm{df}=3(\mathrm{P}=0.88) ; \mathrm{I}^{2}=0 \%$

Test for overall effect: $Z=0.94(P=0.35)$

C

Study or Subgroup log[Odds Ratio] SE Weight IV. Fixed. $95 \% \mathrm{CI}$

Darsaut et al. 2011

Dorfer et al. 2010

Ellis et al. 2013

Fok et al. 2015

Ma et al. 2015

Ma et al. $2017^{40}$

Oulasvirta et al. 2018

$100.0 \% \quad 1.33[0.74,2.40]$

Total $(95 \% \mathrm{Cl})$

Heterogeneity: $\mathrm{Chi}^{2}=8.56$, df $=6(P=0.20) ; \mathrm{I}^{2}=30 \%$

Test for overall effect: $Z=2.92(P=0.004)$

$\begin{array}{llll}-0.6733 & 0.5314 & 14.9 \% & 0.51[0.18,1.45]\end{array}$

$\begin{array}{llll}0.7747 & 0.8502 & 5.8 \% & 2.17[0.41,11.49]\end{array}$

$\begin{array}{llll}0.7839 & 0.5967 & 11.8 \% & 2.19[0.68,7.05]\end{array}$

$\begin{array}{llll}0.8329 & 0.7029 & 8.5 \% & 2.30[0.58,9.12]\end{array}$

$\begin{array}{llll}0.4187 & 0.4256 & 23.2 \% & 1.52[0.66,3.50]\end{array}$

$\begin{array}{llll}1.3297 & 0.5525 & 13.8 \% & 3.78[1.28,11.16]\end{array}$

$\begin{array}{llll}0.9517 & 0.4369 & 22.0 \% & 2.59[1.10,6.10\end{array}$

$100.0 \% \quad 1.82[1.22,2.72]$ $100 \%$

IV. Fixed, $95 \%$ CI

D

Study or Subgroup Odds Ratio Abecassis $\begin{array}{lrrrr}\text { Abecassis et al. } 2016 & -1.1087 & 1.4957 & 4.7 \% & 0.33[0.02,6.19] \\ \text { Anderson et al. 2012 } & 0.6313 & 0.8433 & 14.9 \% & 1.88[0.36,9.82]\end{array}$

Ellis et al. 2013

Ma et al. 2015

Ma et al. $2017^{\text {to }}$

Oulasvirta et al. 2018

Skjoth-Rasmussen et al, 2012

Yang et al. 2016

Total $(95 \% \mathrm{Cl})$

Heterogeneity: $\mathrm{Ch}^{2}=5.89, \mathrm{df}=7(\mathrm{P}=0.55) ; \mathrm{I}^{2}=0 \%$

Test for overall effect: $Z=2.49(P=0.01)$

$\begin{array}{rrrr}0.6313 & 0.8433 & 14.9 \% & 1.88[0.36,9.82] \\ 2.2966 & 0.898 & 13.2 \% & 9.94[1.71,57.78]\end{array}$

$\begin{array}{rrrr}2.2966 & 0.898 & 13.2 \% & 9.94[1.71,57.78] \\ 0.0677 & 0.7627 & 18.3 \% & 1.07[0.24,4.77]\end{array}$

$\begin{array}{rrrr}0.0677 & 0.7627 & 18.3 \% & 1.07[0.24,4.77] \\ 0.6152 & 1.042 & 9.8 \% & 1.85[0.24,14.26]\end{array}$

$\begin{array}{rrrr}0.6152 & 1.042 & 9.8 \% & 1.85[0.24,14.26]\end{array}$

$\begin{array}{llll}0.8838 & 0.7846 & 17.3 \% & 2.42[0.52,11.26]\end{array}$

$\begin{array}{rrrr}0.606 & 1.1756 & 7.7 \% & 1.83[0.18,18.36] \\ 1.3813 & 0.8653 & 14.2 \% & 3.98[0.73,21.70]\end{array}$

$100.0 \% \quad 2.25[1.19,4.26]$

E

Study or Subgroup Je Weight Odds Ratio

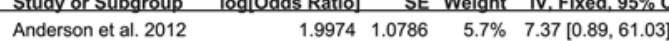

$\begin{array}{lrrrr}\text { Anderson et al. 2012 } & 1.9974 & 1.0786 & 5.7 \% & 7.37[0.89,61.03] \\ \text { Darsaut et al. 2011 } & 0.7275 & 0.5989 & 18.4 \% & 2.07[0.64,6.69]\end{array}$

Ellis et al. 2013

Fok et al. 2015

Ma et al. 2015

Ma et al. 2017

Reitz et al. 2016

Yang et al. 2016 76

$\begin{array}{ll}1.0438 & 0.8018\end{array}$

$10.3 \% 2.84[0.59,13.67]$

$\begin{array}{llll}-0.4005 & 0.643 & 15.9 \% & 0.67[0.19,2.36]\end{array}$

$\begin{array}{llll}0.5306 & 0.6244 & 16.9 \% & 1.70[0.50,5.78]\end{array}$

$\begin{array}{llll}0.3853 & 0.8839 & 8.4 \% & 1.47[0.26,8.31]\end{array}$

$\begin{array}{llll}0.2231 & 0.7281 & 12.4 \% & 1.25[0.30,5.21]\end{array}$

$\begin{array}{llll}-0.5108 & 0.7425 & 12.0 \% & 0.60\end{array}[0.14,2.57]$

Total $(95 \% \mathrm{Cl})$

$100.0 \% \quad 1.46[0.88,2.42]$

Heterogeneity: $\mathrm{Chi}^{2}=6.29, \mathrm{df}=7(\mathrm{P}=0.51) ; \mathrm{I}^{2}=0 \%$

Test for overall effect: $Z=1.48(P=0.14)$

2]

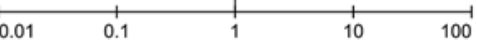

Favours [experimental] ${ }^{1} \quad 10$

\begin{tabular}{|c|c|c|c|c|c|c|c|}
\hline $\begin{array}{l}\text { F } \\
\text { Study or Subgroup }\end{array}$ & log[Odds Ratio] & & Weight & $\begin{array}{l}\text { Odds Ratio } \\
\text { IV. Fixed. } 95 \% \mathrm{Cl}\end{array}$ & $\begin{array}{r}\text { Odds } \\
\text { IV. Fixed }\end{array}$ & $\begin{array}{l}\text { Ratio } \\
\text { d. } 95 \% \mathrm{Cl}\end{array}$ & \\
\hline Ellis et al. 2013 & 2.1054 & 0.6361 & $76.2 \%$ & $8.21[2.36,28.56]$ & & & \\
\hline Fok et al. 2015 & 2.463 & 1.1387 & $23.8 \%$ & $11.74[1.26,109.38]$ & & & \\
\hline Total $(95 \% \mathrm{Cl})$ & & & $100.0 \%$ & $8.94[3.01,26.55]$ & & & \\
\hline $\begin{array}{l}\text { Heterogeneity: } \mathrm{Chi}^{2}= \\
\text { Test for overall effect: }\end{array}$ & $\begin{array}{l}.08, d f=1(P=0.7 \\
=3.94(P<0.000\end{array}$ & 8); $1^{2}=c$ & & & 0.1 & & $100^{\circ}$ \\
\hline
\end{tabular}

G

Study or Subgroup

Abecassis et al. 2016

Darsaut et al. 2011

Dorfer et al. 2010

Nerva et al. 2016

Sanchez-Mejia et al. 2006

Skjoth-Rasmussen et al. 2012

Yang et al. 2016 log[Odds Ratio] SE Weight IV. Fixed. 95\%

Odds Ratio

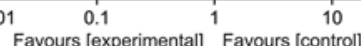

100

Favours [experimental] Favours [control]

Odds Ratio

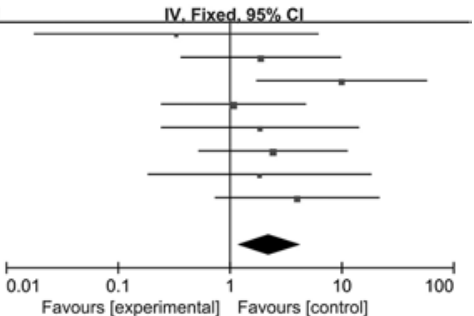

IV. Fixed. $95 \% \mathrm{Cl}$

Total $(95 \% \mathrm{Cl})$

Heterogeneity: $\mathrm{Chi}^{2}=4.60, \mathrm{df}=6(\mathrm{P}=0.60) ; \mathrm{I}^{2}=0 \%$ $\begin{array}{llll}-1.772 & 0.9971 & 4.0 \% & 0.17[0.02,1.20]\end{array}$ $\begin{array}{llll}-0.4155 & 0.2606 & 58.9 \% & 0.66[0.40,1.10]\end{array}$ $\begin{array}{llll}-0.0943 & 0.7992 & 6.3 \% & 0.91[0.19,4.36]\end{array}$ $\begin{array}{llll}-1.6094 & 0.7841 & 6.5 \% & 0.20[0.04,0.93]\end{array}$ $-0.4308 \quad 1.0689 \quad 3.5 \% \quad 0.65[0.08,5.28]$ $\begin{array}{llll}-0.4308 & 1.0689 & 3.5 \% & 0.65[0.08,5.28]\end{array}$ $\begin{array}{llll}-1.1087 & 0.7911 & 6.4 \% & 0.33\end{array}[0.07,1.56]$ $\begin{array}{llll}-0.8675 & 0.5253 & 14.5 \% & 0.42\end{array}[0.15,1.18]$

Test for overall effect: $Z=3.19(P=0.001)$

$100.0 \% \quad 0.53[0.36,0.78]$

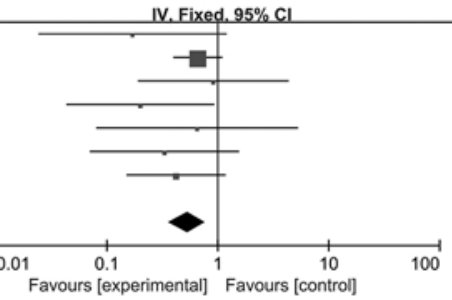

FIG. 4. Forest plots for association of hemorrhagic presentation and other characteristics of AVMs, including AVM size (A), eloquent location (B), deep location (C), infratentorial location (D), associated aneurysm (E), morphology (F), and high SM grade ${ }^{68}(\mathbf{G})$. 
found that the smaller AVMs had 3 times the risk of larger AVMs (OR 2.97, 95\% CI 1.94-4.54, p < 0.00001; Fig. 4A); there was no significant heterogeneity $\left(\mathrm{p}=0.14\right.$ and $\mathrm{I}^{2}=$ $38 \%$ ), and the fixed-effects model was used.

\section{Eloquent AVM Location}

With univariate regression, 4 articles ${ }^{2,11,51,76}$ explored the relationship between eloquent AVM location and hemorrhagic presentation. Because there was no heterogeneity among these articles $\left(\mathrm{p}=0.88\right.$ and $\left.\mathrm{I}^{2}=0 \%\right)$, the fixedeffects model was chosen. We found that there was no association between eloquent location and hemorrhagic presentation (OR 1.33, 95\% CI 0.74-2.40, p = 0.35; Fig. 4B).

\section{AVM Location}

The AVM location was divided into deep/superficial location in 7 articles ${ }^{11,16,17,20,46,47,53}$ and supratentorial/infratentorial location in 8 articles. ${ }^{1,2,17,46,47,53,66,76}$ Before pooling the results, we found that there was no significant heterogeneity in the 2 groups $\left(\mathrm{p}=0.20\right.$ and $\mathrm{I}^{2}=30 \%$, and $\mathrm{p}=0.55$ and $\mathrm{I}^{2}=0 \%$, respectively). The pooled results suggested that AVMs located in deep and infratentorial locations had a higher risk of hemorrhage: OR 1.82 (95\% CI 1.22-2.72, $\mathrm{p}=0.004$; Fig. 4C) and OR 2.25 (95\% CI 1.19-4.26, p = 0.01 ; Fig. 4D), respectively.

\section{Associated Aneurysm}

Eight articles $2,11,17,20,46,47,59,76$ discussed the risk of an associated aneurysm with hemorrhagic presentation. No heterogeneity was found among these articles $(p=0.55$ and $I^{2}=0 \%$ ). Using the fixed-effects model, we found that the associated aneurysm could not increase the risk of hemorrhage in pediatric patients with AVMs (OR 1.46, 95\% CI $0.88-2.42, p=0.14$; Fig. 4E).

\section{Morphology}

Morphology of AVMs included diffuse and compact in 2 articles. ${ }^{17,20}$ The fixed-effects model was used for no heterogeneity $\left(\mathrm{p}=0.78\right.$ and $\left.\mathrm{I}^{2}=0 \%\right)$, and the pooled results suggested that diffuse AVMs had a higher risk of hemorrhage than compact AVMs (OR 8.94, 95\% CI 3.01-26.55, $\mathrm{p}<0.0001$; Fig. 4F).

\section{High SM Grade}

A high SM grade was defined as grade 4 or 5 , and 7 articles $1,11,16,51,62,66,76$ provided the data to calculate the risk of high SM grade with hemorrhagic presentation. The fixedeffects model was used for no heterogeneity $(p=0.60$ and $\left.\mathrm{I}^{2}=0 \%\right)$. The pooled results showed that high SM grade was associated with a lower risk of hemorrhage (OR 0.53 , 95\% CI 0.36-0.78, $\mathrm{p}=0.001$; Fig. 4G).

\section{Publication Bias}

There were 8 factors reported by more than 5 articles. With funnel plots and Begg's test, we found that there was no evidence of publication bias for these factors (Supplemental Fig. 1), including age $(\mathrm{p}=0.064)$, sex $(\mathrm{p}=0.208)$, AVM size $(p=0.343)$, deep venous drainage $(p=0.078)$, deep location $(\mathrm{p}=0.823)$, infratentorial location $(\mathrm{p}=$ $0.426)$, associated aneurysm $(\mathrm{p}=0.274)$, and high SM grade $(\mathrm{p}=0.160)$.

\section{Discussion}

\section{Implications of the Study}

As pediatric and adult patients have different angioarchitecture, clinical presentation, and outcomes of AVMs, they usually present with different hemorrhagic features. ${ }^{17}$ Oulasvirta et al. $^{53}$ reported that pediatric patients with AVMs had a higher tendency to present with hemorrhage than adult patients with AVMs. Hemorrhage increases mortality and causes substantial neurological defects ${ }^{39,42}$ in pediatric patients compared with adult patients. In addition, pediatric patients should be treated more carefully, as the time-dependent features developed gradually and pediatric patients thus have a greater lifetime risk of brain AVM-associated morbidity. ${ }^{42}$ Therefore, identification of factors associated with hemorrhage in pediatric patients could help in the definitive treatment of pediatric AVM. To our knowledge, this is the first meta-analysis assessing the predictors for hemorrhagic presentation in pediatric AVM patients. The probability of hemorrhagic presentation was $63 \%$ $\left(95 \%\right.$ CI $\left.58 \%-69 \%, \mathrm{I}^{2}=72.2 \%\right)$ in pediatric AVM patients. After pooling the results of 15 articles with 1155 patients, we found that the risk of first presentation with hemorrhage was higher in the patients with smaller AVMs, deep venous drainage, a single draining vein, deep cerebral locations, an infratentorial cerebral location, and diffuse AVMs.

In this meta-analysis, the probability of hemorrhagic presentation was higher in pediatric patients with AVMs $(63 \%)$ than in studies of adult patients (30\%-50\%), which is consistent with the findings of many previous studies. ${ }^{22,30}$ Hetts et al. ${ }^{30}$ found that there were significant differences in angioarchitecture of AVMs between pediatric and adult patients. Frontal and temporal AVMs accounted for $40 \%-50 \%$ of AVMs and were more common in adults than in children. AVMs in pediatric patients were more likely located in the basal ganglia, cerebellum, and posterior paracallosal areas, ${ }^{30,71}$ which were associated with higher hemorrhagic presentation of AVMs..$^{14,72}$ In addition, deep venous drainage was more common in pediatric patients than in adult patients and it was also an independent factor for hemorrhage. ${ }^{29,35}$ However, Yang et al. ${ }^{76}$ reported that pediatric patients with AVMs had a lower hemorrhage risk than adult AVM patients. They explained that children tend to not receive clinical attention until their AVMs rupture. One study ${ }^{29}$ reported approximately the same annual hemorrhage risk of 2\%-4\% in pediatric and adult patients with AVMs. Therefore, more studies are needed to evaluate the annual hemorrhage rates for unruptured AVMs and to assess whether there are differences between pediatric and adult AVM patients.

Pooling the results of patients aged $<12$ years and $>$ 12 years for hemorrhagic presentation suggested that age was not associated with hemorrhagic presentation in pediatric AVMs. Skjøth-Rasmussen et al. ${ }^{66}$ showed that the older patients had a significantly higher hemorrhage risk. The inconsistencies might be attributed to the limited number of patients included in this study. However, the mean age of patients in the ruptured AVM group was significantly lower than that in the unruptured AVM group, with a weighted mean difference of -1.53 . This difference suggested that a new threshold of age should be chosen to evaluate this relationship. 
Small nidus size was associated with hemorrhagic presentation, which was consistent with the findings of previous studies. ${ }^{14}$ Spetzler et al. ${ }^{68}$ reported that small AVMs presented with hemorrhage more often than large ones ( $82 \%$ vs $21 \%$, p < 0.001). Although the pathophysiological mechanisms underlying this association remain unclear, they found that smaller AVMs were associated with higher feeding artery pressures upon surgery, as well as larger hematoma sizes; therefore, the most likely reason is that higher feeding artery pressures will increase hemorrhage risk. Despite the high rates of hemorrhagic presentation observed among small AVMs, several prospective studies $^{2,11,53}$ have failed to find an association between AVM size and future hemorrhage. Although Fok et al. ${ }^{20}$ reported that diffuse AVM nidal morphology was associated with higher hemorrhagic presentation, the definition of diffuse AVM was not clear in previous studies. Generally, diffuse $\mathrm{AVM}$ was defined as the presence of significant intervening brain tissue within the AVM nidus. ${ }^{17,20}$ Our analysis found that hemorrhagic presentation of diffuse AVMs was 8 times higher than that of compact AVMs.

The presence of deep venous drainage is an important feature associated with hemorrhage in adult AVMs. Hetts et al.$^{30}$ reported a significantly higher incidence of hemorrhage in pediatric patients with exclusively deep venous drainage compared with adult AVMs. Kellner et al. ${ }^{35}$ identified deep venous drainage, rather than exclusively deep venous drainage, as a predictor for hemorrhagic presentation. Another report ${ }^{17}$ on 135 children with brain AVMs indicated that exclusively deep venous drainage was associated with hemorrhagic presentation, and identified exclusively deep venous drainage to be an independent predictor of hemorrhagic presentation using multivariate analysis. As pediatric AVM angioarchitecture changes over time, the association of deep venous drainage and exclusively deep venous drainage with hemorrhagic presentation becomes complex and remains controversial in children. In our meta-analysis, deep venous drainage $(\mathrm{p}<0.0001)$ and a single draining vein $(p=0.005)$ increased the rate of hemorrhagic presentation. Deep draining veins typically have a smaller diameter than superficial draining veins, as well as a high frequency of stenosis. ${ }^{35}$ Therefore, compared with superficial draining veins, nidi with deep draining veins would have higher pressure, which increases the hemorrhage risk. In addition, the single draining vein has been known as the baseline status of early-stage AVMs, with subsequent veins developing later. ${ }^{17}$ Compared with AVMs with several perinidal vessels, the single draining vein had elevated intranidal stress caused by the increased impedance to blood drainage, which may predispose the AVM to rupture. Thus, deep venous drainage and single venous drainage increased the rate of first presentation with hemorrhage.

Our analysis suggested that the associated aneurysm was not associated with hemorrhagic presentation in pediatric patients with AVMs. Anderson et al. ${ }^{2}$ found no significant difference in the rate of hemorrhage $(p=0.91)$ between isolated AVMs (64\%) and AVM-associated aneurysms $(59 \%)$ in pediatric patients. However, they also found that AVM-associated aneurysms in an arterial location $(91 \%)$ had a higher rate of hemorrhagic presentation compared with isolated AVMs $(\mathrm{p}=0.023)$. In addition, a previous study ${ }^{9}$ found that AVM-associated aneurysms in venous and intranidal locations did not appear to be associated with hemorrhage. The probable explanation is that the flow rate and pressure were higher on the arterial side of the AVM than that in the intranidal location or venous side, which could increase the risk of hemorrhage for arterially based aneurysms. Meanwhile, the arterially based aneurysm would affect the transmural pressure and led to an increased risk of rupture.

The location of AVMs was divided into superficial and deep locations. Some studies ${ }^{46,53}$ found that the deep AVM location was associated with hemorrhagic presentation; the deep AVM location in these studies included the thalamus, brainstem, or basal ganglia. Ding et al. ${ }^{14}$ reported that a significantly higher proportion of ruptured AVMs were at a deep location $(41 \%$ vs $29 \%, p=0.022)$. In our meta-analysis, the deep location was associated with higher hemorrhage risk. Pediatric AVMs are more likely located in the basal ganglia, cerebellar, and posterior paracallosal areas, and AVMs located in a deep location are more likely to have a high frequency of perforating artery supply and deep venous drainage. ${ }^{29}$ Our meta-analysis also indicated that AVMs located in an infratentorial location had a higher risk of hemorrhage, with an OR of 2.25. Khaw et al. ${ }^{36}$ suggested the infratentorial location as an independent predictor of initial and future AVM hemorrhage, and pediatric AVMs in infratentorial locations that often presented with epilepsia could not be found until rupture.

\section{Limitations}

Some limitations exist in this meta-analysis. First, although this meta-analysis only included pediatric AVMs without treatment, more prospective studies that provide the annual hemorrhage rate and factors for initial hemorrhage or recurrent hemorrhage after a long-term followup are needed. Second, the number of articles referring to some factors was limited, such as single feeder and morphology, and these results need more studies to confirm our findings in the future. Moreover, the results of studies referring to age ( $>12$ years) showed a great heterogeneity, and this pooled result needs to be discussed in future high-quality studies. Finally, the definitions of diffuse AVM and compact AVM were not quantitative, and therefore the morphology determined by the researchers might be confounded.

\section{Conclusions}

The hemorrhagic presentation of AVMs was higher in pediatric patients $(63 \%)$ than in adult patients $(30 \%-50 \%)$. The results of this meta-analysis suggested that pediatric AVMs with smaller size $(<3 \mathrm{~cm})$, deep venous drainage, a single feeder, a single draining vein, deep location, infratentorial location, diffuse morphology, and high SM grade (4 or 5) were associated with higher hemorrhagic presentation. Particularly, patients with diffuse AVM have a higher risk of hemorrhagic presentation than other factors and may need active treatment. However, factors including age, sex, draining vein stenosis, and associated aneurysm were not predictors of hemorrhage in pediatric patients with AVMs. 


\section{Acknowledgments}

We thank the professional editors at Editage, a division of Cactus Communications, for the English editing of this paper. This work is supported by science and technology supportive projects of Sichuan Province: "Intracerebral haemorrhage prevention and diagnostic treatment skills" (grant no. 2015SZ0051; Chao You); and "An international, multicenter, large sample randomized controlled trial of supratentorial deep intracerebral haematoma surgery and conservative treatment in adults" (grant no. ZY2016102; Chao You)

\section{References}

1. Abecassis IJ, Nerva JD, Barber J, Rockhill J, Ellenbogen RG, Kim LJ, et al: Toward a comprehensive assessment of functional outcomes in pediatric patients with brain arteriovenous malformations: the Pediatric Quality of Life Inventory. J Neurosurg Pediatr 18:611-622, 2016

2. Anderson RC, McDowell MM, Kellner CP, Appelboom G, Bruce SS, Kotchetkov IS, et al: Arteriovenous malformationassociated aneurysms in the pediatric population. J Neurosurg Pediatr 9:11-16, 2012

3. Bills DC, Rosenfeld JV, Phelan EM, Klug GL: Intracranial arteriovenous malformations in childhood: presentation, management and outcome. J Clin Neurosci 3:220-228, 1996

4. Blamek S, Larysz D, Miszczyk L: Stereotactic linac radiosurgery and hypofractionated stereotactic radiotherapy for pediatric arteriovenous malformations of the brain: experiences of a single institution. Childs Nerv Syst 29:651-656, 2013

5. Blamey SF: Arterio-venous malformations (AVM) hemorrhage in children: the importance of nursing neurological assessment during the acute phase and early recovery. Axone 17:36-41, 1995

6. Börcek AO, Emmez H, Akkan KM, Öcal Ö, Kurt G, Aykol $S$, et al: Gamma Knife radiosurgery for arteriovenous malformations in pediatric patients. Childs Nerv Syst 30:14851492,2014

7. Buis DR, Dirven CM, Lagerwaard FJ, Mandl ES, Lycklama A Nijeholt GJ, Eshghi DS, et al: Radiosurgery of brain arteriovenous malformations in children. J Neurol 255:551-560, 2008

8. Capitanio JF, Gallotti AL, Panni P, Snider S, Scomazzoni F, Mortini P: 204: pediatrics arteriovenous malformations treatment with stereotactic radiosurgery Gamma Knife: our institutional experience. Neurosurgery 63 (Suppl 1):180, 2016

9. Cunha e Sa MJ, Stein BM, Solomon RA, McCormick PC: The treatment of associated intracranial aneurysms and arteriovenous malformations. J Neurosurg 77:853-859, 1992

10. da Costa L, Wallace MC, Ter Brugge KG, O'Kelly C, Willinsky RA, Tymianski M: The natural history and predictive features of hemorrhage from brain arteriovenous malformations. Stroke 40:100-105, 2009

11. Darsaut TE, Guzman R, Marcellus ML, Edwards MS, Tian L, Do HM, et al: Management of pediatric intracranial arteriovenous malformations: experience with multimodality therapy. Neurosurgery 69:540-556, 2011

12. de Castro-Afonso LH, Nakiri GS, Oliveira RS, Santos MV, Santos ACD, Machado HR, et al: Curative embolization of pediatric intracranial arteriovenous malformations using Onyx: the role of new embolization techniques on patient outcomes. Neuroradiology 58:585-594, 2016

13. Dinca EB, de Lacy P, Yianni J, Rowe J, Radatz MW, Preotiuc-Pietro D, et al: Gamma Knife surgery for pediatric arteriovenous malformations: a 25-year retrospective study. J Neurosurg Pediatr 10:445-450, 2012

14. Ding D, Starke RM, Kano H, Mathieu D, Huang PP, Feliciano $C$, et al: International multicenter cohort study of pediatric brain arteriovenous malformations. Part 1: predictors of hemorrhagic presentation. J Neurosurg Pediatr 19:127-135, 2017
15. Ding D, Xu Z, Yen CP, Starke RM, Sheehan JP: Radiosurgery for unruptured cerebral arteriovenous malformations in pediatric patients. Acta Neurochir (Wien) 157:281-291, 2015

16. Dorfer C, Czech T, Bavinzski G, Kitz K, Mert A, Knosp E, et al: Multimodality treatment of cerebral AVMs in children: a single-centre 20 years experience. Childs Nerv Syst 26:681687, 2010

17. Ellis MJ, Armstrong D, Vachhrajani S, Kulkarni AV, Dirks PB, Drake JM, et al: Angioarchitectural features associated with hemorrhagic presentation in pediatric cerebral arteriovenous malformations. J Neurointerv Surg 5:191-195, 2013

18. Englot DJ, Young WL, Han SJ, McCulloch CE, Chang EF, Lawton MT: Seizure predictors and control after microsurgical resection of supratentorial arteriovenous malformations in 440 patients. Neurosurgery 71:572-580, 2012

19. Flickinger JC, Kondziolka D, Lunsford LD, Kassam A, Phuong LK, Liscak R, et al: Development of a model to predict permanent symptomatic postradiosurgery injury for arteriovenous malformation patients. Int J Radiat Oncol Biol Phys 46:1143-1148, 2000

20. Fok EW, Poon WL, Tse KS, Lau HY, Chan CH, Pan NY, et al: Angiographic factors associated with haemorrhagic presentation of brain arteriovenous malformation in a Chinese paediatric population. Hong Kong Med J 21:401-406, 2015

21. Foy AB, Wetjen N, Pollock BE: Stereotactic radiosurgery for pediatric arteriovenous malformations. Neurosurg Clin $\mathbf{N}$ Am 21:457-461, 2010

22. Fullerton HJ, Achrol AS, Johnston SC, McCulloch CE, Higashida RT, Lawton MT, et al: Long-term hemorrhage risk in children versus adults with brain arteriovenous malformations. Stroke 36:2099-2104, 2005

23. Gaballah M, Storm PB, Rabinowitz D, Ichord RN, Hurst RW, Krishnamurthy G, et al: Intraoperative cerebral angiography in arteriovenous malformation resection in children: a single institutional experience. J Neurosurg Pediatr 13:222-228, 2014

24. Galván De la Cruz OO, Ballesteros-Zebadúa P, Moreno-Jiménez S, Celis MA, García-Garduño OA: Stereotactic radiosurgery for pediatric patients with intracranial arteriovenous malformations: variables that may affect obliteration time and probability. Clin Neurol Neurosurg 129:62-66, 2015

25. Griffiths PD, Blaser S, Armstrong D, Chuang S, Humphreys RP, Harwood-Nash D: Cerebellar arteriovenous malformations in children. Neuroradiology 40:324-331, 1998

26. Gross BA, Storey A, Orbach DB, Scott RM, Smith ER: Microsurgical treatment of arteriovenous malformations in pediatric patients: the Boston Children's Hospital experience. J Neurosurg Pediatr 15:71-77, 2015

27. Hanakita S, Koga T, Shin M, Igaki H, Saito N: The long-term outcomes of radiosurgery for arteriovenous malformations in pediatric and adolescent populations. J Neurosurg Pediatr 16:222-231, 2015

28. Hartmann A, Mast H, Mohr JP, Koennecke HC, Osipov A, Pile-Spellman J, et al: Morbidity of intracranial hemorrhage in patients with cerebral arteriovenous malformation. Stroke 29:931-934, 1998

29. Hernesniemi JA, Dashti R, Juvela S, Väärt K, Niemelä M, Laakso A: Natural history of brain arteriovenous malformations: a long-term follow-up study of risk of hemorrhage in 238 patients. Neurosurgery 63:823-831, 2008

30. Hetts SW, Cooke DL, Nelson J, Gupta N, Fullerton H, Amans MR, et al: Influence of patient age on angioarchitecture of brain arteriovenous malformations. AJNR Am J Neuroradiol 35:1376-1380, 2014

31. Higgins J, Green S: Cochrane Handbook for Systematic Reviews of Interventions, version 5.1.0. London: Cochrane Collaboration, 2011

32. Higgins JP, Thompson SG, Deeks JJ, Altman DG: Measuring inconsistency in meta-analyses. BMJ 327:557-560, 2003 
33. Hofmeister C, Stapf C, Hartmann A, Sciacca RR, Mansmann $\mathrm{U}$, terBrugge K, et al: Demographic, morphological, and clinical characteristics of 1289 patients with brain arteriovenous malformation. Stroke 31:1307-1310, 2000

34. Kano H, Kondziolka D, Flickinger JC, Yang HC, Flannery TJ, Awan NR, et al: Stereotactic radiosurgery for arteriovenous malformations, part 2: management of pediatric patients. J Neurosurg Pediatr 9:1-10, 2012

35. Kellner CP, McDowell MM, Phan MQ, Connolly ES, Lavine SD, Meyers PM, et al: Number and location of draining veins in pediatric arteriovenous malformations: association with hemorrhage. J Neurosurg Pediatr 14:538-545, 2014

36. Khaw AV, Mohr JP, Sciacca RR, Schumacher HC, Hartmann A, Pile-Spellman J, et al: Association of infratentorial brain arteriovenous malformations with hemorrhage at initial presentation. Stroke 35:660-663, 2004

37. Kim H, Al-Shahi Salman R, McCulloch CE, Stapf C, Young WL: Untreated brain arteriovenous malformation: patientlevel meta-analysis of hemorrhage predictors. Neurology 83:590-597, 2014

38. Kiran NA, Kale SS, Vaishya S, Kasliwal MK, Gupta A, Sharma MS, et al: Gamma Knife surgery for intracranial arteriovenous malformations in children: a retrospective study in 103 patients. J Neurosurg 107 (6 Suppl):479-484, 2007

39. Kondziolka D, Humphreys RP, Hoffman HJ, Hendrick EB, Drake JM: Arteriovenous malformations of the brain in children: a forty year experience. Can J Neurol Sci 19:40-45, 1992

40. Krings T, Geibprasert S, Terbrugge K: Classification and endovascular management of pediatric cerebral vascular malformations. Neurosurg Clin N Am 21:463-482, 2010

41. Kubalek R, Moghtaderi A, Klisch J, Berlis A, Quiske A, Schumacher M: Cerebral arteriovenous malformations: influence of angioarchitecture on bleeding risk. Acta Neurochir (Wien) 145:1045-1052, 2003

42. Laakso A, Dashti R, Seppänen J, Juvela S, Väärt K, Niemelä $\mathrm{M}$, et al: Long-term excess mortality in 623 patients with brain arteriovenous malformations. Neurosurgery 63:244255,2008

43. Lawton MT, Du R, Tran MN, Achrol AS, McCulloch CE, Johnston SC, et al: Effect of presenting hemorrhage on outcome after microsurgical resection of brain arteriovenous malformations. Neurosurgery 56:485-493, 2005

44. Liu S, Chen HX, Mao Q, You C, Xu JG: Factors associated with seizure occurrence and long-term seizure control in pediatric brain arteriovenous malformation: a retrospective analysis of 89 patients. BMC Neurol 15:155, 2015

45. Lv X, Wu Z, Jiang C, Yang X, Li Y, Sun Y, et al: Angioarchitectural characteristics of brain arteriovenous malformations with and without hemorrhage. World Neurosurg 76:95-99, 2011

46. Ma L, Chen XL, Chen Y, Wu CX, Ma J, Zhao YL: Subsequent haemorrhage in children with untreated brain arteriovenous malformation: higher risk with unbalanced inflow and outflow angioarchitecture. Eur Radiol 27:2868-2876, 2017

47. Ma L, Huang Z, Chen XL, Ma J, Liu XJ, Wang H, et al: Periventricular location as a risk factor for hemorrhage and severe clinical presentation in pediatric patients with untreated brain arteriovenous malformations. AJNR Am J Neuroradiol 36:1550-1557, 2015

48. Ma L, Kim H, Chen XL, Wu CX, Ma J, Su H, et al: Morbidity after hemorrhage in children with untreated brain arteriovenous malformation. Cerebrovasc Dis 43:231-241, 2017

49. Ma X, Tong X, Wu J, Cao Y, Wang S: Seizure control following treatment of brain arteriovenous malformations in pediatric patients. Childs Nerv Syst 32:2387-2394, 2016

50. Mansmann U, Meisel J, Brock M, Rodesch G, Alvarez H, Lasjaunias P: Factors associated with intracranial hemor- rhage in cases of cerebral arteriovenous malformation. Neurosurgery 46:272-281, 2000

51. Nerva JD, Kim LJ, Barber J, Rockhill JK, Hallam DK, Ghodke BV, et al: Outcomes of multimodality therapy in pediatric patients with ruptured and unruptured brain arteriovenous malformations. Neurosurgery 78:695-707, 2016

52. Niazi TN, Klimo P Jr, Anderson RC, Raffel C: Diagnosis and management of arteriovenous malformations in children. Neurosurg Clin N Am 21:443-456, 2010

53. Oulasvirta E, Koroknay-Pál P, Hafez A, Elseoud AA, Lehto $\mathrm{H}$, Laakso A: Characteristics and long-term outcome of 127 children with cerebral arteriovenous malformations. Neurosurgery [epub ahead of print], 2018

54. Pan DH, Kuo YH, Guo WY, Chung WY, Wu HM, Liu KD, et al: Gamma Knife surgery for cerebral arteriovenous malformations in children: a 13-year experience. J Neurosurg Pediatr 1:296-304, 2008

55. Parkhutik V, Lago A, Tembl JI, Vázquez JF, Aparici F, Mainar E, et al: Postradiosurgery hemorrhage rates of arteriovenous malformations of the brain: influencing factors and evolution with time. Stroke 43:1247-1252, 2012

56. Patsopoulos NA, Evangelou E, Ioannidis JP: Sensitivity of between-study heterogeneity in meta-analysis: proposed metrics and empirical evaluation. Int J Epidemiol 37:1148-1157, 2008

57. Potts MB, Sheth SA, Louie J, Smyth MD, Sneed PK, McDermott MW, et al: Stereotactic radiosurgery at a low marginal dose for the treatment of pediatric arteriovenous malformations: obliteration, complications, and functional outcomes. J Neurosurg Pediatr 14:1-11, 2014

58. Provenzale JM: Imaging findings of structural causes of epilepsy in children: a guide for the radiologist in the emergency room. Emerg Radiol 17:479-486, 2010

59. Reitz M, von Spreckelsen N, Vettorazzi E, Burkhardt T, Grzyska U, Fiehler J, et al: Angioarchitectural risk factors for hemorrhage and clinical long-term outcome in pediatric patients with cerebral arteriovenous malformations. World Neurosurg 89:540-551, 2016

60. Reyns N, Blond S, Gauvrit JY, Touzet G, Coche B, Pruvo JP, et al: Role of radiosurgery in the management of cerebral arteriovenous malformations in the pediatric age group: data from a 100-patient series. Neurosurgery 60:268-276, 2007

61. Rubin D, Santillan A, Greenfield JP, Souweidane M, Riina HA: Surgical management of pediatric cerebral arteriovenous malformations. Childs Nerv Syst 26:1337-1344, 2010

62. Sanchez-Mejia RO, Chennupati SK, Gupta N, Fullerton H, Young WL, Lawton MT: Superior outcomes in children compared with adults after microsurgical resection of brain arteriovenous malformations. J Neurosurg 105 (2 Suppl):82-87, 2006

63. Sheth SA, Potts MB, Sneed PK, Young WL, Cooke DL, Gupta N, et al: Angiographic features help predict outcome after stereotactic radiosurgery for the treatment of pediatric arteriovenous malformations. Childs Nerv Syst 30:241-247, 2014

64. Shtaya A, Millar J, Sparrow O: Multimodality management and outcomes of brain arterio-venous malformations (AVMs) in children: personal experience and review of the literature, with specific emphasis on age at first AVM bleed. Childs Nerv Syst 33:573-581, 2017

65. Singhal A, Adirim T, Cochrane D, Steinbok P: Pediatric patients with poor neurological status and arteriovenous malformation hemorrhage: an outcome analysis. J Neurosurg Pediatr 7:462-467, 2011

66. Skjøth-Rasmussen J, Obbekjær T, Birkeland P, Hauerberg J, Juhler M: Multimodality treatment approach in children with cerebral arteriovenous malformation-a survey in the Danish population. Childs Nerv Syst 28:587-592, 2012

67. Soltanolkotabi M, Schoeneman SE, Alden TD, Hurley MC, 
Ansari SA, DiPatri AJ Jr, et al: Onyx embolization of intracranial arteriovenous malformations in pediatric patients. J Neurosurg Pediatr 11:431-437, 2013

68. Spetzler RF, Hargraves RW, McCormick PW, Zabramski JM, Flom RA, Zimmerman RS: Relationship of perfusion pressure and size to risk of hemorrhage from arteriovenous malformations. J Neurosurg 76:918-923, 1992

69. Starke RM, Ding D, Kano H, Mathieu D, Huang PP, Feliciano C, et al: International multicenter cohort study of pediatric brain arteriovenous malformations. Part 2: outcomes after stereotactic radiosurgery. J Neurosurg Pediatr 19:136-148, 2017

70. Tamura N, Hayashi M, Chernov M, Tamura M, Horiba A, Konishi Y, et al: Outcome after Gamma Knife surgery for intracranial arteriovenous malformations in children. $\mathbf{J}$ Neurosurg 117 Suppl:150-157, 2012

71. Tong X, Wu J, Lin F, Cao Y, Zhao Y, Ning B, et al: The effect of age, sex, and lesion location on initial presentation in patients with brain arteriovenous malformations. World Neurosurg 87:598-606, 2016

72. Turjman F, Massoud TF, Viñuela F, Sayre JW, Guglielmi G, Duckwiler G: Correlation of the angioarchitectural features of cerebral arteriovenous malformations with clinical presentation of hemorrhage. Neurosurgery 37:856-862, 1995

73. Wells G, Shea B, O'Connell D, Peterson J, Welch V, Losos M: Newcastle-Ottawa Scale (NOS) for Assessing the Quality of Nonrandomised Studies in Meta-Analyses. Ottawa: Ottawa Hospital Research Institute, 2011 (http://www. ohri.ca/programs/clinical_epidemiology/oxford.asp) [Accessed October 2, 2018]

74. Wong ST, Fong D: Ruptured brain arteriovenous malformations in children: correlation of clinical outcome with admission parameters. Pediatr Neurosurg 46:417-426, 2010

75. Yang H, Deng Z, Yang W, Liu K, Yao H, Tong X, et al: Predictive factors of postoperative seizure for pediatric patients with unruptured arteriovenous malformations. World Neurosurg 105:37-46, 2017

76. Yang W, Anderson-Keightly H, Westbroek EM, Caplan JM, Rong X, Hung AL, et al: Long-term hemorrhagic risk in pediatric patients with arteriovenous malformations. J Neurosurg Pediatr 18:329-338, 2016

77. Yang W, Caplan JM, Ye X, Wang JY, Braileanu M, Rigamonti D, et al: Racial associations with hemorrhagic presentation in cerebral arteriovenous malformations. World Neurosurg 84:461-469, 2015

78. Yang W, Liu A, Hung AL, Braileanu M, Wang JY, Caplan $\mathrm{JM}$, et al: Lower risk of intracranial arteriovenous malformation hemorrhage in patients with hereditary hemorrhagic telangiectasia. Neurosurgery 78:684-693, 2016
79. Yang W, Westbroek EM, Anderson-Keightly H, Caplan JM, Rong X, Hung AL, et al: Male gender associated with posttreatment seizure risk of pediatric arteriovenous malformation patients. Neurosurgery 80:899-907, 2017

80. Yen CP, Monteith SJ, Nguyen JH, Rainey J, Schlesinger DJ, Sheehan JP: Gamma Knife surgery for arteriovenous malformations in children. J Neurosurg Pediatr 6:426-434, 2010

81. Yeon JY, Shin HJ, Kim JS, Hong SC, Lee JI: Clinico-radiological outcomes following gamma knife radiosurgery for pediatric arteriovenous malformations. Childs Nerv Syst 27:1109-1119, 2011

82. Zeiler FA, Janik MK, McDonald PJ, Kaufmann AM, Fewer D, Butler J, et al: Gamma Knife radiosurgery for pediatric arteriovenous malformations: a Canadian experience. Can J Neurol Sci 43:82-86, 2016

83. Zheng T, Wang QJ, Liu YQ, Cui XB, Gao YY, Lai LF, et al: Clinical features and endovascular treatment of intracranial arteriovenous malformations in pediatric patients. Childs Nerv Syst 30:647-653, 2014

\section{Disclosures}

The authors report no conflict of interest concerning the materials or methods used in this study or the findings specified in this paper.

\section{Author Contributions}

Conception and design: Jiang, Ai, Ye. Acquisition of data: Ai, Ye. Analysis and interpretation of data: Jiang, Ai, Ye. Drafting the article: Ai. Critically revising the article: Xu, You. Reviewed submitted version of manuscript: $\mathrm{Xu}$, You. Approved the final version of the manuscript on behalf of all authors: Jiang. Statistical analysis: Jiang, Ai, Ye.

\section{Supplemental Information \\ Online-Only Content}

Supplemental material is available with the online version of the article.

Supplemental Fig. 1. https://thejns.org/doi/suppl/10.3171/2018. 9.PEDS18262.

\section{Correspondence}

Yan Jiang: West China Hospital of Sichuan University, Sichuan, China.jipin_li@163.com. 